\title{
Evaluation of Telecommunications Electromagnetic Compatibility with the Use of Three-Dimensional Modeling Technology
}

\author{
Tatiana Sergeevna Abbasova ${ }^{1}$, Natalya Petrovna Sidorova ${ }^{1}$, Natalya Nikolaevna Teodorovich ${ }^{1} \&$ Elshan \\ Maggeram Oglu Abbasov ${ }^{1}$ \\ 1 The State-Funded Educational Institution of Higher Education Moscow region University of Technology, \\ Korolev city, Moscow region, Russia \\ Correspondence: Tatiana Sergeevna Abbasova, The State-Funded Educational Institution of Higher Professional \\ Education Moscow region University of Technology, Korolev city, Moscow region, Gagarina street, 42, Russia. \\ Tel: 8-906-716-2082. E-mail: abbasova_univer@mail.ru
}

\author{
Received: March 5, 2016 \\ Accepted: May 5, 2016 \\ Online Published: July 27, 2016 \\ doi:10.5539/mas.v10n10p224 \\ URL: http://dx.doi.org/10.5539/mas.v10n10p224
}

The research is financed by (University of Technology).

\begin{abstract}
Three-dimensional modeling is used as a new way of presenting information about the electromagnetic compatibility of telecommunications infrastructure equipment. We analyzed the problem of modeling of electromagnetic noise waves equipment and communication channels of telecommunications monitoring systems. Conducted the analysis of control problems of telecommunications equipment electromagnetic compatibility. Described the real conditions of installing the electronic equipment in computational complexes. Using tools of 3D-modeling was formed cable route, taking into account the suppression of noise waves, placement of telecommunication devices and checking whether the threshold value of cable susceptibility for all options of its gasket between devices was transcended. It is proposed to study the behavior of electromagnetic fields in the cable lines with shielding cable, electrostatic discharge, providing set of measures for electromagnetic compatibility, to convert created in a three-dimensional environment model in a specialized settlement package. The prospects of the use 4D-, 5D-, 6D-simulation for analysis of the implementation of projects, reduction of operating and electromagnetic compatibility costs.
\end{abstract}

Keywords: telecommunications infrastructure, electromagnetic compatibility, computer simulation

\section{Introduction}

Replacing of outdated monitoring systems associated with the processes of integration of operators through the acquisition of small telecommunications networks with diverse equipment as well as the emergence of new features of modern monitoring systems for interactive management. During the process of monitoring systems centralizing great importance has the quality of data center functioning (Computing Complex (CC)). CC is characterized by a high degree of electronic devices integration. Problems of improving the noise immunity and electromagnetic compatibility (EMC) of the equipment for the control and monitoring of telecommunications networks are relevant. Analysis of methods and means of evaluating the EMN exposure on the CC equipment and telecommunications and communication channels [Moroz, Beluchenko, Samarov and Strenakuk, 2014; Artyushenko and Abbasova, 2014; Abbasova, Artuschenko and Samarov, 2014; Moroz, Poserenin, Stroitelev \& Vasilyev, 2014] showed that the reliable assessment of noise immunity requires the development of new models of EMN exposure evaluation on the investigated objects.

One of the promising directions for the development of telecommunications systems and related equipment of monitoring systems taking into account EMC, as well as the implementation of new telecommunications modules in the old modules, is the use of 3D-images to simulate the electromagnetic noise (EMN).

To control EMN is conducted the analysis of the information flow parameters of distortion in the conditions of EMN exposure. A distinctive feature of EMN impact on the CC equipment and telecommunication infrastructure is not the physical destruction of the components and communication channels, but the violation of the logical integrity of information, transmitted over these lines of communication and processed by computing complex 
[Artyushenko and Abbasova, 2014; Artuschenko, Samarov, Golubev, Shchikanov \& Kochetkov, 2015; Beluchenko, Zinoviev, Strenakuk \& Vatutin, 2014; Vatutin, Semyonov \& Shavrin, 2014]. Assessment of the stability of $\mathrm{CC}$ and telecommunication infrastructure when they exposed to electromagnetic noise includes determination of the parameters and subsequent assessment of the EMN impact on the performance of individual components and assemblies of $\mathrm{CC}$ and the monitoring system in general. Analysis of the problems with theoretical and experimental methods for the study of EMN exposure on the CC equipment and telecommunication infrastructure and methods of assessment the noise immunity revealed the following patterns:

1) Existing numerical methods for calculating the efficiency of screening and methods of calculation of voltages and currents that affect the $\mathrm{CC}$ equipment and telecommunication infrastructure require improvement and development of engineering techniques on their basis that can be used on the $\mathrm{CC}$ design stages to determine the main parameters of influence.

2) It is necessary to assess the impact of electromagnetic noise on telecommunication equipment. It is impossible to obtain reliable data only by analytical methods due to the diversity of the principles of their construction and operation, as well as complexity of accounting the real electromagnetic relations with the surrounding space.

3) In the study of immunity of the CC equipment and telecommunications infrastructure should be taken into account existing methods and remedies.

\section{Method}

As a method of modeling based on international and Russian experience offered the three-dimensional models of noise waves and network infrastructure, in which the noise waves are measured. Simulation tasks: preliminary assessment of EMN levels at any point of the CC or communication channel, as well as drawing a conclusion about the possibility of installing a new system, the introduction of a new module or wiring. Using these estimates in the monitoring system we may reduce the level of EMN by generating the necessary control signal that initiate specific actions on the use of the selected method of controlling the operating noise. The real simulated device or the transmission environment are three-dimensional and, as a rule, not uniform in their electric and magnetic properties. Therefore, to obtain reliable results, it is often necessary to solve the three-dimensional problems. On the example of three-dimensional model of the network infrastructure can be carried out detailed planning work for ensuring the EMC of telecommunication equipment.

\subsection{Methods of modeling noise waves in electrical communication channels of the telecommunication infrastructure}

Nowadays in high-speed telecommunications networks for production purposes, as well as in ground-based space infrastructure are used not only optical fiber, but also shielded and unshielded electrical cables. This is due to the fact that not on all sites of the network infrastructure is advisable to use fiber optic cables. For example, to connect the workstations of employees are used the communication channels with a bandwidth of not more than $100 \mathrm{Mbit} / \mathrm{s}$, so as the transmission environment are used unshielded UTP cables of 6th category (patch cords), shielded cables STP of 7th category (communication channels before switchboards that combine subnets). Between switchboards and for connection to servers are used fiber optic cables. The proposed accounting model of EMN of electric channels in telecommunication infrastructure is different from the well-known by the following criteria:

- $\quad$ detection of devices, instruments, workstations, connected by cable;

- rendering application for numerical simulation of the process, providing the analysis and interpretation of the calculation results (not only the value of the noise waves themselves, but also the cable mounting parameters, taking into account the suppression of noise and location of telecommunication devices);

- the possibility of further work with computer model in different modeling environments (For example, Compass 3D, 3D-Maxwell);

- $\quad$ perception and interpretation of visual images by the developer of the cable system.

\subsection{Participant (Subject) Characteristics}

The study involved researchers from the (Moscow State) University of Technology. Research interests: information and communication technologies.

\subsection{Tools of Analytical, Geometric and Three-Dimensional Modeling}

To develop the three-dimensional model of noise waves in the cables we should analyze the interaction of two wires in a magnetic field using a magnetic field theory [Buchholz, 1961; Govorkov, 1968; Demirchyan, 1974]. 
In accordance with the law of total current:

$$
\int_{0}^{2 \pi r} H d s=I
$$

where $H$ is the magnetic field around the wire through which a current $I$ flows.

At a distance $L$ from the current-carrying wire, the magnetic field will be equal to:

$$
H=I / 2 \pi l,[A / m] .
$$

The magnetic field generated around the current-carrying wire 1 includes a wire 2, as shown in Figure 1.



Figure 1. Three-dimensional geometric interpretation of the magnetic coupling between «working» chains of wires 2,3 , and noise waves source 1

The mechanism of magnetic coupling that occurs between wires describe using Faraday's law:

$$
\int E d s=-\frac{d \Phi}{d t}=-L \frac{d I}{d t}=U
$$

where $E$ is the electric field intensity, $[\mathrm{V} / \mathrm{m}] ; U$ is the voltage induced in the circuit formed by the wires $2-3$;

$$
F=\int B d S
$$

where $B$ is the magnetic flux crossing the loop area of the $S$, formed by wires $2-3 ; B=\mu N ; \mu$ is magnetic permeability of the environment.

Define the voltage induced in the circuit $2-3$ (see Figure 1). Assuming that the length of the wires 2 and 3 is equal to $L$. Using the results of [Demirchyan, 1974] and omitting intermediate calculations, taking $h=h_{23}$, substituting in equation (4) the limits of integration, we can write:

$$
F=\int_{l_{12}}^{l_{12}+l_{23}} B L d l=\mu L \int_{l_{12}}^{l_{12}+l_{23}} H d l=\frac{\mu L I}{2 \pi} \int_{l_{12}}^{l_{12}+l_{23}} \frac{d l}{l}=\frac{\mu L I}{2 \pi} \operatorname{Lg}\left(\frac{l_{12}+l_{23}}{l_{12}}\right) .
$$

Then

$$
U=-\frac{d F}{d t}=-\frac{\mu L}{2 \pi} \operatorname{Lg}\left(\frac{l_{12}+l_{23}}{l_{12}}\right) \frac{d I}{d t}=-\mu L f \operatorname{Lg}\left(\frac{l_{12}+l_{23}}{l_{12}}\right),
$$


where

$$
\left|\frac{d I}{d t}\right|=\left|\frac{d}{d t}(I \cos \omega t)\right|=\omega I=2 \pi f I
$$

Analysis of equation (6) shows that the induced voltage in the circuit will increase with the increasing of magnetic permeability $\mu$, the chain length of $L, I$ amperage and frequency $f$. When you remove wires $2-3$ from the source of noise waves, induced voltage will decrease. After simple algebraic transformations, using the results of [Demirchyan, 1974], we can write that transitive attenuation or coupling coefficient $K_{c}$ between the source of noise waves and «working» wires can be defined as:

$$
K_{c}=\frac{U_{2}}{U_{1}}=\frac{Z_{22} \mu L f}{\left(Z_{12}+Z_{22}+j \omega L_{2}\right)\left(Z_{11}+Z_{21}+j \omega L_{1}\right)} \cdot \lg \left(\frac{l_{12}+l}{l_{12}}\right),
$$

where $U_{1}$ is the voltage of power (noise waves) source of line $1 ; U_{2}$ is the voltage induced in the «working» line 2 because of magnetic coupling; $Z_{11}, Z_{21}$ are the internal resistance of the voltage source of noise waves and «working» line respectively; $Z_{12}, Z_{22}$ are the full impedance of the noise source circuit and «working» line respectively; $L_{1}, L_{2}$ are the inductance of the noise source circuit and «working» line respectively.

At low and high frequencies, under certain assumptions, the equation (8) can be represented as:

with $\omega L_{\text {ind }}<<2 Z$

$$
K_{c}=\frac{U_{2}}{U_{1}}=\frac{\mu L f}{4 Z} \lg \left(\frac{l_{12}+l_{23}}{l_{12}}\right) ;
$$

with $\omega L_{\text {ind }}>>2 Z$, excluding phase

$$
K_{c}=\frac{U_{2}}{U_{1}}=\frac{Z \mu L}{4 \pi^{2} f L_{\text {ind }}^{2}} \operatorname{Lg}\left(\frac{l_{12}+l_{23}}{l_{12}}\right),
$$

where $L_{\text {ind }}=L_{1}=L_{2} ; Z \approx Z_{1} \approx Z_{2}$.

Calculation with the use of formulas (6)...(10) allows you to define the permissible values in terms of reduction the magnetic coupling between the "working" wire and the source of noise waves:

- permissible voltage reduction of noise source or current in the circuit 1 ;

- allowable decreasing in line length $L$ and (or) the distance between the wires $h$;

- allowable separation of circuits 1 and 2, so that for the equation (6) the following conditions were completed:

$$
l_{12}>>l_{23} \text { and } \operatorname{Lg}\left[\left(l_{12}+l_{23}\right) / l_{12}\right] \rightarrow \operatorname{Ln} 1=0 ;
$$

allowable decreasing in the frequency of the transmitted signals;

the possibility of screening the «working» wire by magnetic shield with high permeability.

$\mathrm{CC}$ that usually located in a small area is a consolidated computational infrastructure with a high degree of electronic hardware integration. According to the standard MIL-STD-461F, measurements of the radiation (intensities of electric or magnetic fields) are produced at a distance of $1 \mathrm{~m}$ from the tested technical device (TTD). At closer distances from the TTD calibration characteristics of antennas deteriorate, and accurately assess the intensity of the radiated field is not possible. In real conditions, under the installation of electronic facilities in the CC, the gap, separating this equipment, much less than $1 \mathrm{~m}$ (often it is about $5 \mathrm{~cm}$ ). At very short distances from the generating source field can differ significantly from the measured and increase due to the neighboring electronic and technical equipment. The installed facilities will be exposed to radiation more than calculated.

EMN simulation in $\mathrm{CC}$ is reduced to the alternate modeling of electronic devices that work on some area (including devices of communication channels), and subsequent combining them into a system for the parameters determination of intensity of their joint field at any point of the modeled $\mathrm{CC}$ segment. Alternate modeling of each electronic device separately consists of its provisional replacement by an equivalent source, 
depending on the values of the current and voltage in the electrical circuits. Preliminary replacement includes the selection of the equivalent source parameters, based on the coincidence of the dynamics of the distribution of the field intensity at a certain distance from the source. Further obtained equivalent source is represented as a 3D-model in a software environment.

\section{Results}

3D-model is constructed taking into account the geometrical dimensions of the real electronic device. Figure 2 shows the result of EMN simulation of telecommunication unit of $\mathrm{CC}$ with three simulators of electronic devices that generate electromagnetic noise. EMN simulation results can be represented by 3D-models at different times for the visual displaying of the EMC performance.

For the determination of cable routes boundaries is conducted 3D modeling, which allows to specify the location of the boundaries of the cable when cabling under EMC requirements. Figure 3 shows how, with the applying of various tools of software application «Cables and plaits 3D» (specialized application for KOMPAS-3D), cable route is formed, taking into account the placement of devices (fittings, blocks) and checking whether the threshold value of cable susceptibility for all options of its gasket between devices was transcended. Three-dimensional modeling can also be used for the gasket of an shielded electrical cable, taking into account the impact of the electromagnetic noise waves penetration through the screens.

To study the behavior of electromagnetic fields in cable routes (for example, during the processes of shielding cable, electrostatic discharge, ensuring a set of EMC measures) we can use created in KOMPAS-3D environment model and move it to ANSYS Maxwell environment (leading software for 2D and 3D simulation of electromagnetic fields).

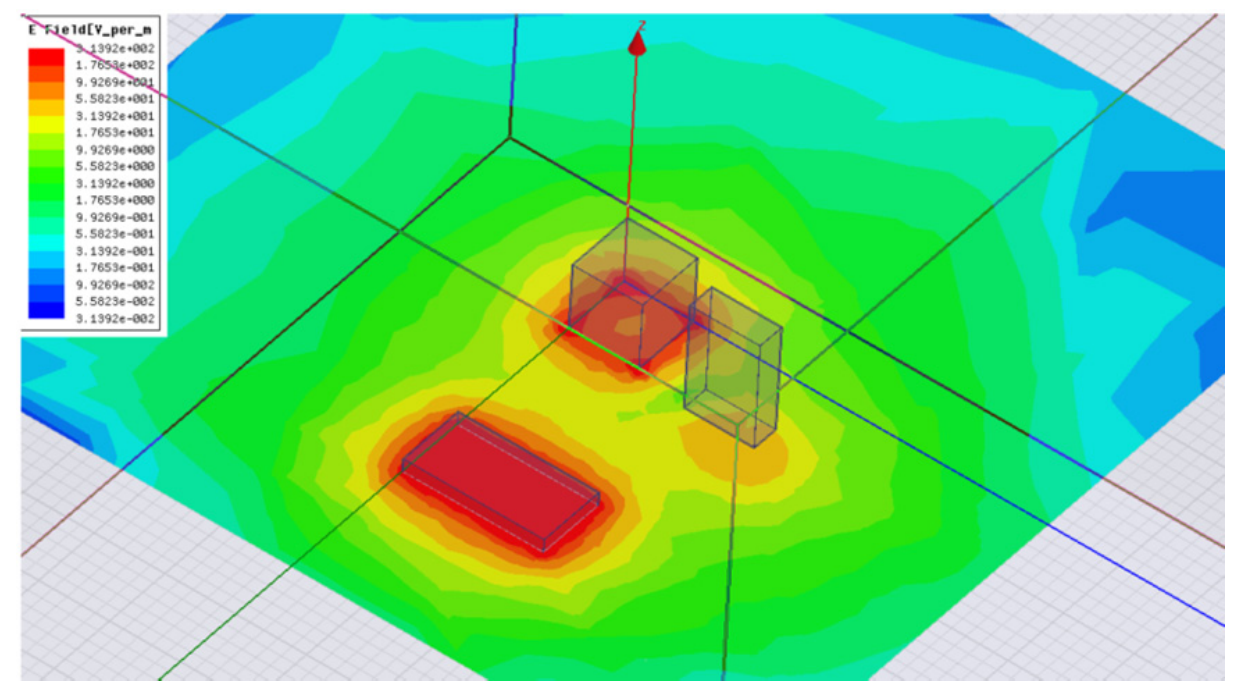

Figure 2. The result of the telecommunication facilities modeling with three simulators of electronic devices

Three-dimensional models of cables and plaits are based on the number and diameter of conductors, extending along the routes; taking into account the fillets in the track turning points and automatically calculated the conditional diameter of the cable or plait branch. With the help of three-dimensional modeling of CC infrastructure the equipment can be distributed for the adjacent modules, depending on the purpose of using (business center, room for cable entry, distribution point of horizontal subsystem, etc.) in accordance with ANSI/TIA/EIA-942 Telecommunications Infrastructure Standard for standard Data Centers (Figure 4). This will improve the reliability and physical security of equipment.

Thus, obtained spatial 3D-models of assessment the EMN of electronic devices in the CC module, electrical communication channels of telecommunications infrastructure, $\mathrm{CC}$ equipment distribution through modules. On the basis of the EMN evaluation are developed protective measures, aimed at bringing the EMN in accordance with the requirements of the digital equipment. 


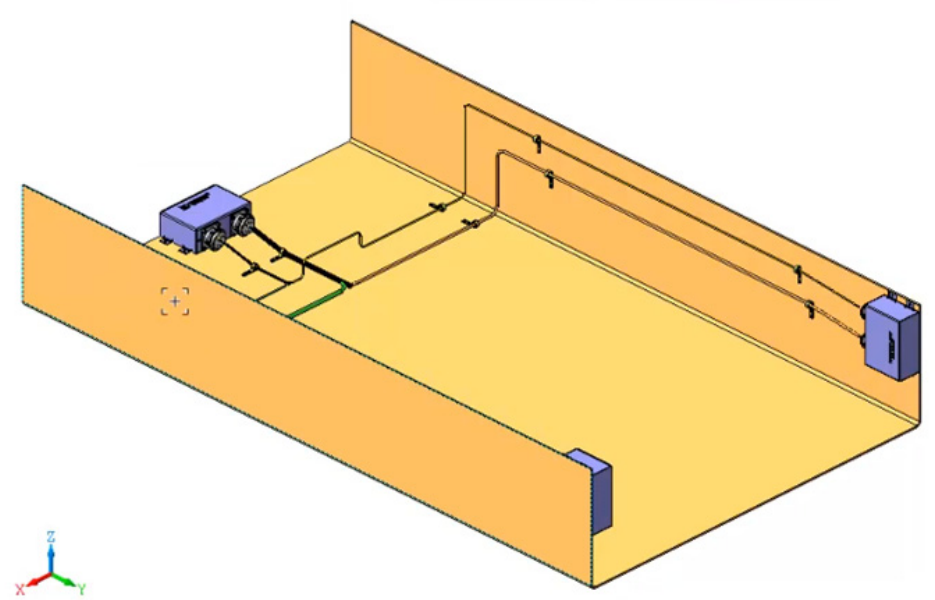

Figure 3. The results of cable routes modeling in the program KOMPAS-3D

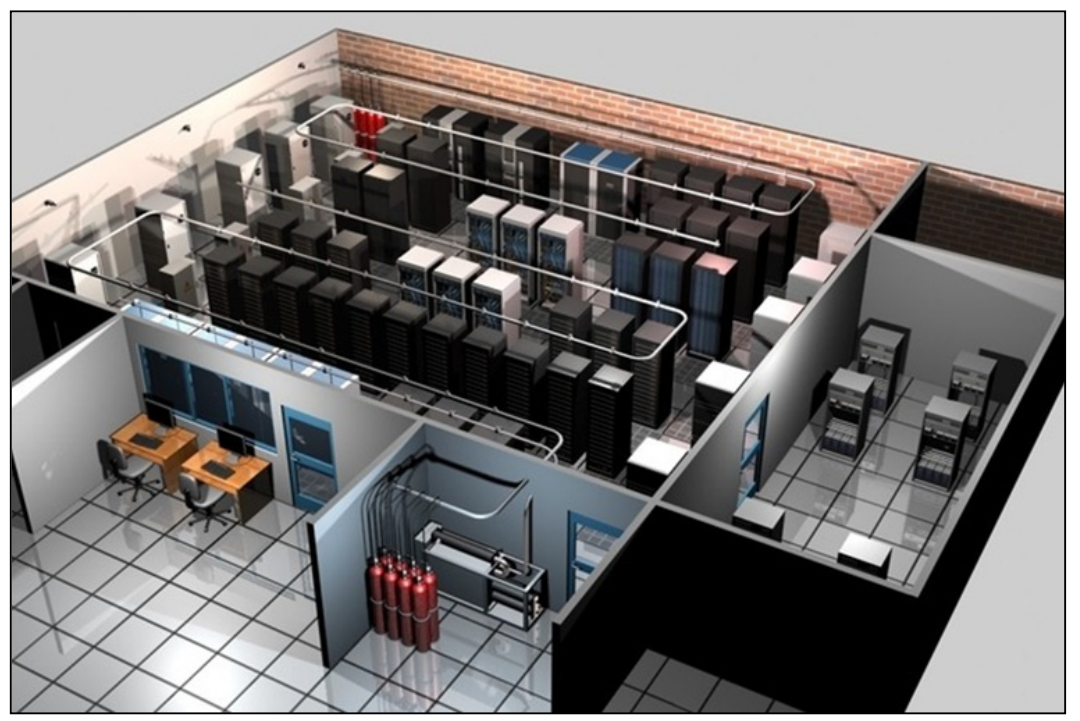

Figure 4. 3D-model of the integrated computational complex

In the Results section, summarize the collected data and the analysis performed on those data relevant to the discourse that is to follow. Report the data in sufficient detail to justify your conclusions. Mention all relevant results, including those that run counter to expectation; be sure to include small effect sizes (or statistically nonsignificant findings) when theory predicts large (or statistically significant) ones. Do not hide uncomfortable results by omission. Do not include individual scores or raw data with the exception, for example, of single-case designs or illustrative examples. In the spirit of data sharing (encouraged by APA and other professional associations and sometimes required by funding agencies), raw data, including study characteristics and indivldual effect sizes used in a meta -analysis, can be made available on supplemental online archives.

\section{Discussion}

For reduction the operational costs, including costs for ensuring the EMC, it is advisable to use three-dimensional models created in an software environment of $\mathrm{N}$-dimensional modeling $(4 \mathrm{D}, 5 \mathrm{D}, 6 \mathrm{D})$.

4D-model associated with the calendar and the network schedule of the project implementation of the new module (modernization of the existing module) in telecommunications infrastructure. 5D-model reflects the visual detailing of the project cost. $6 \mathrm{D}$-model visually reflects the situation at the facility at any time, visual «planned vs. actual» progress of the project analysis. After the completion of the implementation (modernization) process constructed models can be used as a constantly actualized data base containing information on the 
channels of communication and telecommunication infrastructure equipment.

\section{Conclusions}

The proposed three-dimensional model of EMN accounting for equipment and communication channels of the telecommunications infrastructure is different from the well-known by following criteria: detection of devices, instruments, workstations, connected by cable; rendering application for numerical modeling of the process, providing the analysis and interpretation of the results of calculations (not only the magnitude of noise itself, but also the cable mounting options, taking into account the suppression of noise and location of telecommunication devices); the possibility of further work with computer models in different software environments (Compass 3D, 3D (2D) -Maxwell, 4D, 5D, 6D); perception and interpretation of visual images by the developer of the cable system. Appealing to the three-dimensional modeling techniques provides a number of important practical advantages: improve the conditions of design and administration of CC cable system.

\section{Acknowledgments}

The authors express their gratitude to Doctor of Technical Sciences, Professor of the University of Technology (Russia) Vladimir Mikhailovich Artyushenko for reasonable and principled criticism in conduction of the research and writing the scientific article.

\section{References}

Abbasova, T. S., Artyushenko, V. M., \& Samarov, K. L. (2014). Modern Methods of Processing of Video Information and Evaluating the Quality of Streaming Video Perception. Biosci., Biotech. Res. Asia, 11(Nov. Spl. Edn.), 265-268. ISSN: 0973-1245. http://dx.doi.org/10.13005/bbra/1473.

Artuschenko, V. M., Samarov, K. L., Golubev, A. P., Shchikanov, A. Y., \& Kochetkov, A. S. (2015). Modeling of Correlated Two-Dimensional Non-Gaussian Noises. Modern Applied Science, 9(8), 309-319. ISSN 1913-1844; E-ISSN 1913-1852. Published by Canadian Center of Science and Education. http://dx.doi.org/10.5539/mas.v9n8p300

Artyushenko, V. M., \& Abbasova, T. S. (2014). Increasing Noise Immunity of Electric Communication Channels in High-speed Telecommunication Systems. Biosci., Biotech. Res. Asia, 11(Nov. Spl. Edn.), 277-279. ISSN: 0973-1245. http://dx.doi.org/10.13005/bbra/1475.

Beluchenko, I. M., Zinoviev, V. N, Strenakuk, Y. V., \& Vatutin, V. M. (2014). Analysis of the Decoder's Operation at Correcting Single Errors Using the Triple Code. Biosci., Biotech. Res. Asia, 11(Nov. Spl. Edn.), 207-210.ISSN: 0973-1245. http://dx.doi.org/10.13005/bbra/1463.

Buchholz, G. (1961). Calculation of electric and magnetic fields. M.: Energia. p. 712.

Demirchyan, K. S. (1974). Modeling of magnetic fields. L.: Energy. p. 385.

Govorkov, V. A. (1968). Electric and magnetic fields. M.: Energia. p. 487.

Moroz A. P., Poserenin S. P., Stroitelev V. N., \& Vasilyev N. A. (2014). The Analisys of The On-line Analytical Processesing Methods For The Information Processing. Biosci., Biotech. Res. Asia, Vol. 11 (Nov. Spl. Edn.), 341-343. ISSN: 0973-1245. DOI: http://dx.doi.org/10.13005/bbra/1484.

Moroz, A. P., Beluchenko, I. M., Samarov K. L. \& Strenakuk Y. V. (2014). Singularities of the Data Anti-jam in Telecommunication Systems Using the New Methods of Encryption. Biosci., Biotech. Res. Asia, 11(Nov. Spl. Edn.), 133-137. ISSN: 0973-1245. http://dx.doi.org/10.13005/bbra/1450.

Vatutin V. M., Semyonov A. B., \& Shavrin, S. S. (2014). The Computerized Simulation of the Neuro-fuzzy System for Recognizing the Parameters of the Geographically Distributed Systems Equipment. Biosci., Biotech. Res. Asia, 11(Nov. Spl. Edn.), 301-305. ISSN: 0973-1245. http://dx.doi.org/10.13005/bbra/1475

\section{Copyrights}

Copyright for this article is retained by the author(s), with first publication rights granted to the journal.

This is an open-access article distributed under the terms and conditions of the Creative Commons Attribution license (http://creativecommons.org/licenses/by/4.0/). 